\title{
Comparison of Analgesic Effect between Gabapentin and Diclofenac on Post-Operative Pain in Patients Undergoing Tonsillectomy
}

\author{
Mario I Ortiz ${ }^{1, *}$, Luis C Romero-Quezada ${ }^{1}$ \\ ${ }^{1}$ Medicine Academic Area, Health Sciences Institute at the Autonomous University of Hidalgo State, Pachuca, Hidalgo, Mexico \\ ${ }^{*}$ Corresponding author: Mario I Ortiz, Medicine Academic Area, Health Sciences Institute at the Autonomous University of Hidalgo State, Pachuca, Hidalgo, Mexico. Tel: $+52-$ \\ 7717172000, Fax:+52-7717172000. Email:, E-mail: mario_i_ortiz@hotmail.com \\ Received: January 25, 2013; Accepted: January 30, 2013
}

Keywords: Gabapentin; Diclofenac

\section{Dear Editor,}

We have examined attentively the latest manuscript by Yeganeh Mogadam et al. entitled "Comparison of Analgesic Effect between Gabapentin and Diclofenac on Post-Operative Pain in Patients Undergoing Tonsillectomy" available in Archives of Trauma Research (1). Ninety patients were included to receive $20 \mathrm{mg} / \mathrm{kg}$ oral gabapentin $(\mathrm{n}=$ $30), 1.0 \mathrm{mg} / \mathrm{kg}$ rectal diclofenac $(\mathrm{n}=30)$ or placebo $(\mathrm{n}=$ 30) preoperatively. Pain was evaluated postoperatively at 2, 6,12 and 24 hours. Authors concluded that gabapentin and diclofenac reduced postoperative pain and opioid consumption without any obvious side effects.

Diclofenac is highly bound to serum proteins ( $\geq 99.5 \%$ ) and has a relatively low volume of distribution (0.12 to $0.17 \mathrm{~L} / \mathrm{kg}$ ) (2,3). Diclofenac easily penetrates the synovial fluid and crosses the placenta $(2,3)$. However, diclofenac does not easily cross the blood-brain barrier. It has been proved that the diclofenac concentrations in cerebrospinal fluid is $8.22 \%$ compared to its value in plasma (4). There are reports wherein the anti-inflammatory and antinociceptive effect of diclofenac cannot be directly explained by circulating concentrations in humans (2, 3). Therefore, it has been suggested that diclofenac effects are mainly mediated via a local action in target tissues. In the present study, it is probable that diclofenac produced prostaglandins inhibition to oropharynx level and with that decreasing the sensitization of nociceptors at this level. On the other hand, although gabapentin is a structural analogue of GABA, which does not cross the blood-brain barrier, gabapentin penetrates into the central nervous system (5). Gabapentin binds to plasma pro- teins and it is not metabolized by nor inhibits hepatic enzymes that are responsible for the metabolism of other drugs (6). It is well known that gabapentin has analgesic and antihyperalgesic effects and is used in the control of clinical pain $(6,7)$. In this sense, the properties of gabapentin have been attributed to an action on the central and peripheral nervous system. Therefore, it is suggested that the gabapentin-induced analgesic effect in the present study was mediated by pain modulation at local level and on the descending pathways. In the present study, children and adult patients were evaluated together. It has been suggested that children may need moderately higher dosages of gabapentin to reach plasma concentrations comparable with those found in adults (8). Likewise, differences have been found in the volume of distribution, Cmax and clearance of diclofenac between children and adults $(9,10)$. Therefore, all the participants should have been evaluated separately to obtain a more consistent result.

In the case of the pain intensity, authors stated on the background section that the post-operative pain in patients undergoing tonsillectomy may be as high as 70 in the scale of visual analog scale. However, the pain intensity in the present study was very low (from 1.53 to 3.43 ). Therefore, the effects of gabapentin and diclofenac appear very scarce or nil. On the other hand, the comparison of pain intensity in the three groups in the manuscript seems wrong. Whereas authors state that the patients' pain in gabapentin group was significantly less than that of the placebo group $(\mathrm{P}<0.05)$ and the mean pain intensity in diclofenac group at 6 hours after the surgery 
was significantly less than that of the placebo group (P $<0.05$ ). Data shows that the pain intensities at 6,12 and 24 hours in the gabapentin and diclofenac groups were bigger than in the placebo group. Only the pain intensities at 2 hours in the treated groups were lower than in the placebo group. This mistake was probably a measurement mistake, made by the authors.

\section{Acknowledgements}

None declared.

\section{Authors' Contribution}

Ortiz MI and Romero-Quezada LC were involved in writing this manuscript.

\section{Financial Disclosure}

There is no financial interest.

\section{Funding/Support}

No funding or supports were received for this letter.

\section{References}

1. Yeganeh Mogadam A, Fazel MR, Parviz S. Comparison of Analgesic Effect Between Gabapentin and Diclofenac on Post-Operative
Pain in Patients Undergoing Tonsillectomy. Arch Trauma Res. 2012;1(3):108-11.

2. Davies NM, Anderson KE. Clinical pharmacokinetics of diclofenac. Therapeutic insights and pitfalls. Clin Pharmacokinet. 1997;33(3):184-213.

3. Fowler PD, Shadforth MF, Crook PR, John VA. Plasma and synovial fluid concentrations of diclofenac sodium and its major hydroxylated metabolites during long-term treatment of rheumatoid arthritis. Eur J Clin Pharmacol. 1983;25(3):389-94.

4. Zecca L, Ferrario P, Costi P. Determination of diclofenac and its metabolites in plasma and cerebrospinal fluid by high-performance liquid chromatography with electrochemical detection. JChromatogr. 1991;567(2):425-32.

5. McLean Michael J, Gidal Barry E. Gabapentin dosing in the treatment of epilepsy. Clinical therapeutics. 2003;25(5):1382-1406.

6. Bockbrader HN, Wesche D, Miller R, Chapel S, Janiczek N, Burg er P. A comparison of the pharmacokinetics and pharmacodynamics of pregabalin and gabapentin. Clin Pharmacokinet. 2010;49(10):661-9.

7. Chou R. ACP Journal Club. Review: Gabapentin reduces some types of chronic neuropathic pain more than placebo in adults. Ann Intern Med. 2011;155(2):JC1-8.

8. Gatti G, Ferrari AR, Guerrini R, Bonanni P, Bonomi I, Perucca E. Plasma gabapentin concentrations in children with epilepsy: influence of age, relationship with dosage, and preliminary observations on correlation with clinical response. Ther Drug Monit. 2003;25(1):54-60.

9. Korpela R, Olkkola KT. Pharmacokinetics of intravenous diclofenac sodium in children. Eur JClin Pharmacol. 1990;38(3):293-5.

10. Murphy DB, Kavanagh P, O'Connor P, Sherrin P, McNamara S, O'Hare B. Pharmacokinetic profile of rectally administered diclofenac sodium in children undergoing adenotonsillectomy. Paediatr Anaesth. 2000;10(6):694-5. 